\title{
CONTRIBUIÇÃO PARA O CONHECIMENTO DA DIETA DE CORUJAS (AVES: STRIGIFORMES) NO SUL DO BRASIL
}

\author{
Renata Brentano ${ }^{1}$, Luiz Liberato Costa Corrê $a^{1,2}$,Douglas Ribeiro da Silva ${ }^{1}$ \& Maria Virginia \\ $\operatorname{Petry}^{1 *}$
}

${ }^{1}$ Universidade do Vale do Rio dos Sinos, Programa de Pós-Graduação em Biologia, Laboratório de Ornitologia e Animais Marinhos, Av. Unisinos, no 950, Bairro Cristo Rei, CEP 93022-750, São Leopoldo, RS, Brasil.

${ }^{2}$ Universidade do Vale do Taquari, Programa de Pós-Graduação em Ambiente e Desenvolvimento, Rua Avelino Talini, no 171, CEP 95900-000, Lajeado, RS, Brazil.

Emails: renata.brentano2014@gmail.com; lc_correa@yahoo.com.br; douglasnhrs@gmail.com; vpetry@unisinos.br (*autor correspondente)

Resumo: Nós analisamos as presas consumidas por quatro espécies de corujas que ocorrem no estado do Rio Grande do Sul, Brasil. As amostras foram obtidas a partir da coleta de uma egagrópila de A. clamator $(\mathrm{N}=1)$ e 12 carcaças de Strigiformes atropelados (Tyto furcata: $\mathrm{N}=5$; Megascops sp.: $\mathrm{N}=4$; Bubo virginianus: $\mathrm{N}=2$; Asio clamator: $\mathrm{N}=1$ ) encontrados em rodovias do estado entre 2001 e 2019. Nós contabilizamos 40 itens alimentares representados por seis classes animais: Arachnida, Chilopoda, Insecta, Amphibia, Aves e Mammalia. Entre os itens ingeridos por T. furcata estavam a rã-manteiga (Leptodactylus latrans) e o rato-doméstico (Mus musculus). Megascops sp. predou principalmente artrópodes e uma pomba-debando (Zenaida auriculata). Os indivíduos de A. clamator predaram um roedor e Coleoptera, enquanto B. virginianus predou apenas roedores. A análise das presas consumidas por Strigiformes ampliou o conhecimento sobre a dieta do grupo no sul do Brasil.

Palavras-chave: Asio clamator; Bubo virginianus; Megascops sp.; Tyto furcata.

CONTRIBUTION TO THE KNOWLEDGE OF THE OWLS DIET (AVES: STRIGIFORMES) IN SOUTHERN BRAZIL. We analyzed the prey consumed by four species of owls that occur in Rio Grande do Sul state, Brazil. Samples were obtained from a pellet by Asio clamator $(\mathrm{N}=1)$ and 12 Strigiformes road killed carcasses (Tyto furcata: $\mathrm{N}=5$; Megascops sp.: $\mathrm{N}=4$; Bubo virginianus: $\mathrm{N}=2$; Asio clamator: $\mathrm{N}=1$ ) found on highways of the state between 2001 and 2019. We counted 40 food items represented by six animal classes: Arachnida, Chilopoda, Insecta, Amphibia, Aves and Mammalia. Tyto furcata fed mainly on the butter frog (Leptodactylus latrans) and the house mouse (Mus musculus). Megascops sp. preyed mainly arthropods and an eared dove (Zenaida auriculata). The A. clamator preyed a rodent and Coleoptera, while both $B$. virginianus preyed only rodents. The analysis of prey consumed by Strigiformes increased knowledge about the group diet in southern Brazil.

Keywords: Asio clamator; Bubo virginianus; Megascops sp.; Tyto furcata.

Grande parte das espécies de corujas apresentam hábitos noturnos e crepusculares, o que dificulta sua visualização e o estudo de seus hábitos alimentares (Sick 1997). A dieta dessas aves inclui variados artrópodes e vertebrados, como anfíbios, répteis, aves e mamíferos de pequeno e médio 
porte (Tomazzoni et al. 2004, Motta-Junior 2006, Baladrón \& Bó 2017). Dessa maneira, o grupo desempenha importante papel biológico (Horváth et al. 2018). No Brasil, os estudos que abordam a dieta de Strigiformes concentram-se na região sul e sudeste (Motta-Junior \& Alho 2000, MottaJunior 2002, Scheibler \& Christoff 2004, MottaJunior 2006, Lemos et al. 2015). No estado do Rio Grande do Sul existem estudos prévios sobre a dieta da suindara Tyto furcata (Strigiformes: Tytonidae) (Scheibler \& Christoff 2004), jacurutu Bubo virginianus (Strigiformes: Strigidae) (Tomazzoni et al. 2004), coruja-buraqueira Athene cunicularia (Strigiformes: Strigidae) (Zilio 2006, Garcia et al. 2008) e corujinha-do-sul Megascops sanctaecatarinae (Strigiformes: Strigidae) (Zilio et al. 2018).
As corujas são vítimas frequentes de atropelamentos (Novelli et al. 1988, Carvalho et al. 2014, Corrêa et al. 2017) ao cruzarem rodovias (Bencke \& Bencke 1999, Arnold et al. 2019) ou ao tentarem se alimentar de carcaças de outros animais atropelados (Erritzoe et al. 2003, Fischer et al. 2018). A análise de conteúdos estomacais de aves atropeladas pode fornecer importantes informações sobre a composição de sua alimentação (Ramos et al. 2011). Diante desse contexto, o objetivo do presente estudo foi analisar conteúdos estomacais de Strigiformes vítimas de atropelamento em rodovias do estado do Rio Grande do Sul, Brasil, ampliando o conhecimento sobre a dieta desse grupo.

Foram analisadas uma egagrópila (i.e., material não digerido) de Asio clamator coletada no

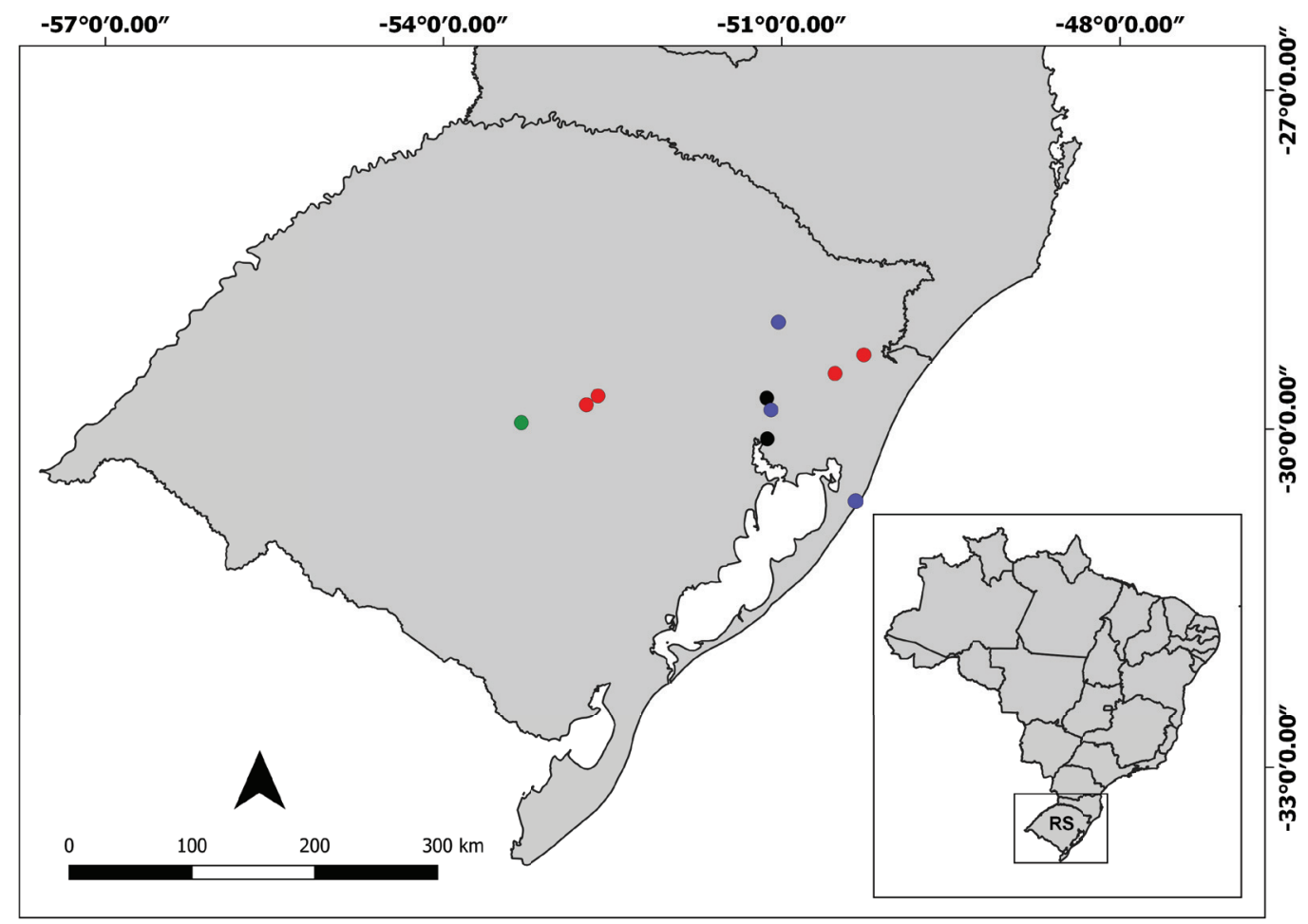

Figura 1. Locais de coleta dos Strigiformes atropelados em rodovias do estado do Rio Grande do Sul, Brasil. As diferentes cores representam as quatro espécies de corujas: Tyto furcata em azul, Megascops sp. em vermelho, Asio clamator em preto e Bubo virginianus em verde. Os números representam os municípios de coleta: 1 - São Leopoldo; 2 - São Marcos; 3 - Mostardas; 4 - Vale do Sol; 5 - São Francisco de Paula; 6 - Candelária, 7 - Restinga Seca; 8 - Estância Velha; 9 - Porto Alegre.

Figure 1. Strigiformes sample collection sites in Rio Grande do Sul state, Brazil. The different colors represent the four species of owls: Tyto furcata in blue, Megascops sp. in red, Asio clamator in black and Bubo virginianus in green. The numbers represent the municipalities of collection: 1 - São Leopoldo; 2 - São Marcos; 3 - Mostardas; 4 - Vale do Sol; 5 - São Francisco de Paula; 6 - Candelária, 7 - Restinga Seca; 8 - Estância Velha; 9 - Porto Alegre. 
Tabela 1. Strigiformes atropelados e coletados em rodovias do estado do Rio Grande do Sul, Brasil, entre 2001 e 2019. (-) Sem informação. * Egagrópila.

Table 1. Strigiformes trampled and collected on highways in Rio Grande do Sul state, Brazil, between 2001 and 2019. (-) Without information. * Pellet.

\begin{tabular}{|c|c|c|c|c|}
\hline Espécie & Sexo & Município & Coordenadas & Estação \\
\hline Tyto furcata & - & São Leopoldo & $\begin{array}{c}29^{\circ} 45^{\prime} 18,74 " \mathrm{~S} \\
51^{\circ} 9^{\prime} 5,53^{\prime \prime} \mathrm{O}\end{array}$ & Verão \\
\hline Tyto furcata & macho & São Marcos & $\begin{array}{l}28^{\circ} 58^{\prime} 14,84^{\prime \prime} \mathrm{S} \\
51^{\circ} 4^{\prime} 19,33^{\prime \prime} \mathrm{O}\end{array}$ & Primavera \\
\hline Tyto furcata & - & Mostardas & $\begin{array}{c}30^{\circ} 34^{\prime} 30,23 " \mathrm{~S} \\
50^{\circ} 25^{\prime} 3,53^{\prime \prime} \mathrm{O}\end{array}$ & Primavera \\
\hline Tyto furcata & - & - & - & - \\
\hline Tyto furcata & - & - & - & - \\
\hline Megascops sp. & fêmea & Vale do Sol & $\begin{array}{l}29^{\circ} 36^{\prime} 14,31^{\prime \prime} \mathrm{S} \\
52^{\circ} 41^{\prime} 17,40 \text { "O }\end{array}$ & Primavera \\
\hline Megascops sp. & - & São Francisco de Paula & $\begin{array}{l}29^{\circ} 26^{\prime} 33,71^{\prime \prime} \mathrm{S} \\
50^{\circ} 34^{\prime} 47,86^{\prime \prime} \mathrm{O}\end{array}$ & Outono \\
\hline Megascops sp. & fêmea & Candelária & $\begin{array}{l}29^{\circ} 40^{\prime} 52,09^{\prime \prime} \mathrm{S} \\
52^{\circ} 47^{\prime} 32,25 ” \mathrm{O}\end{array}$ & Outono \\
\hline Megascops sp. & - & São Francisco de Paula & $\begin{array}{l}29^{\circ} 16^{\prime} 22,23 " \mathrm{~S} \\
50^{\circ} 19^{\prime} 21,344^{\prime \prime} \mathrm{O}\end{array}$ & Outono \\
\hline Bubo virginianus & macho & Restinga Seca & $\begin{array}{l}29^{\circ} 49^{\prime} 44,18^{\prime \prime} \mathrm{S} \\
53^{\circ} 22^{\prime} 13,71^{\prime \prime} \mathrm{O}\end{array}$ & Inverno \\
\hline Bubo virginianus & macho & Restinga Seca & $\begin{array}{l}29^{\circ} 49^{\prime} 16,05^{\prime \prime} \mathrm{S} \\
53^{\circ} 22 \times 26,76^{\prime \prime} \mathrm{O}\end{array}$ & Inverno \\
\hline Asio clamator & - & Estância Velha & $\begin{array}{l}29^{\circ} 38^{\prime} 58,80^{\prime \prime} \mathrm{S} \\
51^{\circ} 11^{\prime} 13,555^{\prime \prime O}\end{array}$ & Outono \\
\hline Asio clamator* & - & Porto Alegre & $\begin{array}{c}30^{\circ} 0 \prime 38,30 " \mathrm{~S} \\
51^{\circ} 11^{\prime} 19,64 " \mathrm{O} \\
\end{array}$ & Outono \\
\hline
\end{tabular}

município de Porto Alegre em 2019 (Tabela 1; Figura 1) e 12 carcaças de Strigiformes atropelados entre 2001 a 2017 (T. furcata: $\mathrm{N}=5$; Megascops sp.: $\mathrm{N}=4 ;$ B. virginianus: $\mathrm{N}=2 ;$ A. clamator: $\mathrm{N}=1$ ). Os espécimes coletados foram acondicionados em sacos plásticos e armazenados em caixa de isopor com gelo e encaminhados ao Laboratório de Ornitologia e Animais Marinhos (LOAM) da Universidade do Vale do Rio dos Sinos (autorização SISBIO $n^{\circ}$ 56689-1). Em laboratório as amostras foram triadas e os itens alimentares separados, quantificados e determinados ao menor nível taxonômico possível. Para determinação dos insetos seguimos Triplehorn \& Johnson (2011). Os demais itens foram comparados ao acervo ornitológico disponível no Museu de Zoologia da Universidade do Vale do Rio dos Sinos ou enviados a especialistas.

Dos 12 estômagos analisados, 11 apresentaram conteúdo com indícios de predação recente, e o estômago de umindivíduo de T. furcata encontravase vazio. Foram contabilizados 40 itens alimentares distribuídos em seis classes animais: Arachnida, Chilopoda, Insecta, Amphibia, Aves e Mammalia (Tabela 2). Nos estômagos de T. furcata, Megascops sp. e A. clamator foram encontrados invertebrados e vertebrados, e nos estômagos de $B$. virginianus foram encontrados somente vertebrados (Tabela 2).

A análise do conteúdo estomacal das quatro espécies de corujas revelou considerável diversidade de itens alimentares, incluindo desde artrópodes como aranhas e insetos, até pequenos vertebrados como anfíbios, aves e mamíferos. Tais resultados corroboram outros estudos sobre Strigiformes que reportam uma dieta generalista (Motta-Junior \& Alho 2000, Motta-Junior 2006). A presença de um anfíbio no estômago de $T$. furcata na primavera corrobora estudos prévios 
Tabela 2. Composição de presas ingeridas por quatro espécies de corujas a partir de 12 conteúdos estomacais de indivíduos atropelados e uma egagrópila coletados no estado do Rio Grande do Sul, Brasil, entre 2001 e 2019. NI = não identificado.

Table 2. Composition of prey ingested by four species of owls from 12 stomach contents of trampled individuals and a pellet collected in Rio Grande do Sul, Brazil, between 2001 and 2019. NI =Without information.

\begin{tabular}{|c|c|c|c|c|c|c|c|}
\hline Classe & Ordem & Família & Espécie & $\begin{array}{c}\text { Tyto } \\
\text { furcata } \\
(\mathrm{N}=5)\end{array}$ & $\begin{array}{c}\text { Megascops } \\
\text { sp. } \\
(\mathrm{N}=4) \\
\end{array}$ & $\begin{array}{c}\text { Bubo } \\
\text { virginianus } \\
(\mathrm{N}=2)\end{array}$ & $\begin{array}{c}\text { Asio } \\
\text { clamator } \\
(\mathrm{N}=2)\end{array}$ \\
\hline Arachnida & Araneae & Lycosidae & Hogna sp. & & 4 & & \\
\hline Chilopoda & Scolopendromorpha & Scolopendridae & $\begin{array}{l}\text { Scolopendra } \\
\text { sp. }\end{array}$ & & 1 & & \\
\hline Insecta & Orthoptera & Acrididae & NI & & 10 & & \\
\hline Insecta & Orthoptera & Tetigoniidae & NI & & 6 & & \\
\hline Insecta & Coleoptera & Scarabeaidae & NI & & 2 & & \\
\hline Insecta & Coleoptera & Cerambycidae & NI & & 1 & & \\
\hline Insecta & NI & $\mathrm{NI}$ & NI & 2 & 1 & & 1 \\
\hline Insecta & Lepidoptera & NI & NI & 1 & 4 & & \\
\hline Amphibia & Anura & Leptodactylidae & $\begin{array}{l}\text { Leptodactylus } \\
\text { latrans }\end{array}$ & 1 & & & \\
\hline Aves & Columbiformes & Columbidae & $\begin{array}{l}\text { Zenaida } \\
\text { auriculata }\end{array}$ & & 1 & & \\
\hline Mammalia & Rodentia & Muridae & $\begin{array}{c}\text { Mus } \\
\text { musculus }\end{array}$ & 1 & & 1 & \\
\hline \multirow[t]{2}{*}{ Mammalia } & Rodentia & NI & NI & 1 & & 1 & 1 \\
\hline & Total & & & 6 & 30 & 2 & 2 \\
\hline
\end{tabular}

que consideram a predação desse táxon um comportamento oportunista, quando roedores, sua principal presa, está em menor disponibilidade no ambiente (Motta-Junior \& Alho 2000). Enquanto a predação de uma ave por Megascops sp. no outono pode ter ocorrido devido à menor disponibilidade de insetos nesse período (Triplehorn \& Johnson 2011, Vieira et al. 2015).

Sabe-se que T. furcata se alimenta de insetos, anfíbios, répteis e aves, porém, é especializada na captura de roedores, incluindo uma grande variedade desse grupo em sua alimentação (Cherem et al. 2018, Mancini et al. 2019). Estudos demostram que essa coruja tende a variar a dieta conforme a disponibilidade das presas nos locais de forrageio (Teta et al.2012, Lemos et al.2015, Zúñiga et al. 2018). Em ambientes pouco antropizados, espécies nativas são as mais consumidas por essa ave (Zúñiga et al. 2018), enquanto que em ambientes urbanos e rurais, o rato-doméstico Mus musculus (Rodentia: Muridae) está entre suas presas mais frequentes (Teta et al. 2012, Cherem et al. 2018), representando cerca de $80 \%$ das presas consumidas por essa coruja na região do Vale do Taquari, estado do Rio Grande do Sul (Scheibler \& Christoff 2004). A abundância de anfíbios na dieta da espécie também parece variar conforme a região geográfica. Em Santana do Araguaia, no estado do Pará, os anfíbios representaram menos de $2 \%$ da dieta de T. furcata (Rocha et al. 2011), enquanto que em Buenos Aires, na Argentina, esse grupo representou $50 \%$ dos itens predados (Teta et al. 2012). Leptodactylus sp. (Anura: Leptodactylidae) foi reportado na dieta de T. furcata na Argentina (Fernández et al. 2009) e no estado do Pará (Rocha et al. 2011), podendo constituir um importante recurso alternativo para a espécie.

Existem poucasinformações sobre a composição alimentar de Megascops sp. para o Brasil. Nossos resultados demonstraram o consumo de artrópodes por Megascops sp., principalmente Orthoptera. No estado de São Paulo, Orthoptera representou $41 \%$ das presas de M. choliba, seguido por Arachnida (11\%) (Motta-Junior 2002), enquanto na Colômbia, Orthoptera correspondeu a $43,5 \%$ da dieta dessa espécie (Delgado 2007). Além de invertebrados, 
M. choliba pode consumir pequenos vertebrados, como anfíbios da família Hylidae e marsupiais do gênero Gracilinanus spp. (Didelphimorphia: Didelphidae) (Motta-Junior 2006, Vieira et al. 2015). Porém, a predação de vertebrados foi mais frequente na dieta de M. sanctaecatarinae, onde representaram $23 \%$ dos itens consumidos e são considerados um importante recurso alimentar para essa espécie de coruja (Zilio et al. 2018).

A dieta de B. virginianus é composta principalmente por pequenos e médios vertebrados, principalmente aves e mamíferos (Tomazzoni et al. 2004, Formoso et al. 2012), embora ocasionalmente presas grandes como o zorrilho Conepatus chinga (Carnivora: Mephitidae) também possam fazer parte da dieta da espécie (Anza \& Zilio 2015). Além disso, espécies exóticas parecem contribuir como um importante recurso alimentar para essa espécie de coruja, a exemplo da garça-vaqueira Bubulcus ibis (Pelecaniformes: Ardeidae) no estado do Tocantins (Dornas \& Pinheiro 2007), da lebre europeia Lepus europaeus (Lagomorpha: Leporidae) na Argentina e no estado do Rio Grande do Sul (Trejo et al. 2005, Peters et al. 2009), e do coelho-europeu Oryctolagus cuniculus (Lagomorpha: Leporidae) no Chile (Jaksić \& Marti 1984).

Vertebrados também são as principais presas de A. clamator (Pautasso 2006, Baladrón \& Bó 2017). Na Argentina, pequenos mamíferos representaram $87 \%$ das presas na dieta da espécie, dos quais $78 \%$ eram roedores (Baladrón \& Bó 2017). Entre as principais espécies encontradas na dieta dessa coruja estão espécies introduzidas como $M$. musculus e o rato-preto Rattus rattus (Rodentia: Muridae) (Delgado et al. 2005). No presente trabalho, além do roedor $M$. musculus, também foram contabilizadas 18 sementes, mas que não foram incluídas como parte da dieta dessa coruja. Uma vez que sementes fazem parte da dieta de roedores (Le Roux et al. 2002), as sementes encontradas no estômago provavelmente foram ingeridas pelo individuo predado por A. clamator. Apesar do baixo número amostral, a análise do conteúdo estomacal dos indivíduos atropelados forneceu informações complementares que permitem ampliar o conhecimento sobre a dieta de corujas no sul do Brasil.

\section{AGRADECIMENTOS}

Somos gratos a César Rodrigo dos Santos e Antônio Coimbra de Brum pelas coletas. Agradecemos ao Dr. Everton Nei Lopes Rodrigues pela determinação de Arachnidae e Chilopoda e a MSc. Heloisa Allgayer pela contribuição na identificação dos roedores. Este estudo foi financiado em parte pela Coordenação de Aperfeiçoamento de Pessoal de Nível Superior.

\section{REFERÊNCIAS}

Anza, J., \& Zilio, F. 2015. Molina's hog-nosed skunk as prey of the great horned owl: predation or opportunist scavenging. Revista Brasileira de Ornitologia, 23(4), 377--379.

Arnold, E. M., Hanser, S. E., Regan, T., Thompson, J., Lowe, M., Kociolek, A., \& Belthoff, J. R. 2019. Spatial, road geometric and biotic factors associated with Barn Owl mortality along an interstate highway. Ibis, 161(1), 147--161. DOI: 10.1111/ibi.12593

Baladrón, A.V., \& Bó, M. S. 2017. Dieta anual del lechuzón orejudo (Asio clamator) em el limite austral de su distribución. Ornitologia Neotropical, 28(1), 51--56.

Bencke, G. A., \& Bencke, C. S. C. 1999. The potential importance of road deaths as cause of mortality for large forest owls in southern Brazil. Cotinga, 11(1), 79--80.

Carvalho, N. C. D., Bordignon, M. O., \& Shapiro, J. T. 2014. Fast and furious: a look at the death of animals on the highway MS-080, Southwestern Brazil. Iheringia. Série Zoologia, 104(1), 43--49. DOI: $10.1590 / 1678-4766201410414349$

Cherem, J. J., Hadler, P., Stutz, N. S., \& Pardinãz, U. F. J. 2018. Pequenos mamíferos (Didelphimorphia, Chiroptera e Rodentia) em egagropilos de Tyto furcata (coruja-das-igrejas) (Aves, Tytonidae) do sul do Brasil. Biotemas, 31(3), 43--58. DOI: 10.5007/2175-7925.2018v31n3p43

Corrêa, L. L. C., Silva, D. E., Oliveira, S. V., Finger, J. V. G., Santos, C. R., \& Petry, M. V. 2017. Vertebrate Road kill survey on a highway in southern Brazil. Acta Scientiarum. Biological Sciences, 39(2), 219-225. DOI: $10.4025 /$ actascibiolsci.v39i2.33788

Delgado, C. A. 2007. Dieta del currucutú Megascops choliba (Strigidae) en la ciudad de Medellín, Colombia. Boletín SAo, 17(2), 114--117. 
Delgado, C. A., Pulgarín P. C., \& Calderón, D. 2005. Análisis de egagrópilas del búho rayado (Asio clamator) en la ciudad de Medellín. Ornitología Colombiana, 3(1), 100--103.

Dornas, T., \& Pinheiro, R. T. 2007. Predação de Opisthocomus hoazin por Spizaetus ornatus e de Bubulcus ibis por Bubo virginianus em Tocantins, Brasil. Revista Brasileira de Ornitologia, 15(4), 601--604.

Erritzoe, J., Mazgajski, T. D., \& Rejt, Ł. 2003. Bird casualties on European roads-a review. Acta Ornithologica, 38(2), 77--94.

Fernández, F. J., Moreira, G., Ferraro, D., \& Santis, L. 2009. Presas consumidas por la lechuza de campanario (Tyto alba) en la localidad de Olavarría, Buenos Aires: un caso de elevada batracofagia. Nuestras Aves, 54(1), 20--21.

Fischer, W., Godoi, R. F., \& Paranhos Filho, A. C. 2018. Roadkill records of reptiles and birds in Cerrado and Pantanal landscapes. Check List, 14(5), 845. DOI: 10.15560/14.5.845

Formoso, A. E., Pablo, T., \& Germán, C. 2012. Food habits of the Magellanic Horned Owl (Bubo virginianus magellanicus) at Southernmost Patagonia, Argentina. Journal of Raptor Research, 46(4), 401--406. DOI: 10.3356/JRR-1222.1

Garcia, S. A., Cristofoli, S. I., Bastian, A. M. S., Fraga, E. D., \& Sander, M. 2008. Sobre mortandade de anfíbios em área de reprodução de Corujaburaqueira, Athene cunicularia (Molina, 1782) em São Leopoldo-RS. Biodiversidade Pampeana, 6(2), 11--13.

Horváth, A., Morvai, A., \& Horváth, G. F. 2018. Foodniche pattern of the Barn Owl (Tyto alba) in intensively cultivated agricultural landscape. Ornis Hungarica, 26(1), 27--40. DOI: 10.1515/ orhu-2018-0002

Jaksić, F. M., \& Marti, C. D. 1984. Comparative food habits of Bubo owls in Mediterranean-type ecosystems. The Condor, 86(3), 288--296. DOI: $10.2307 / 1366997$

Le Roux, V., Chapuis, J. L., Frenot, Y., \& Vernon, P. 2002. Diet of the house mouse (Mus musculus) on Guillou Island, Kerguelen archipelago, Subantarctic. Polar Biology, 25(1), 49--57. DOI: 10.1007 /s003000100310

Lemos, H. M., Silva, C. A. O., Patiu, F. M., \& Gonçalves, P. R. 2015. Barn Owl pellets (Aves: Tyto furcata) reveal a higher mammalian richness in the Restinga de Jurubatiba National Park, Southeastern Brazil. Biota Neotropica, 15(2), 1--9. DOI: 10.1590/1676-06032015012114

Mancini, M. C., Roth, P. R. O., Brennand, P. G., Aguilar, J. M. R. E., \& Rocha, P. A. 2019. Tyto furcata (Tytonidae: Strigiformes) pellets: tools to access the richness of small mammals of a poorly known Caatinga area in northeast Brazil. Mammalia, 83(4), 390--398. DOI: 10.1515/ mammalia-2018-0017

Motta-Junior, J. C. 2002. Diet of breeding Screechowls (Otus choliba) in southeastern Brazil. Journal of Raptor Research, 36(4), 332--334.

Motta-Junior, J. C. 2006. Relações tróficas entre cinco Strigiformes simpátricas na região central do Estado de São Paulo, Brasil. Revista Brasileira de Ornitologia, 14(4), 359--377.

Motta-Junior, J. C., \& Alho, C. J. R. 2000. Ecologia alimentar de Athene cunicularia e Tyto alba (Aves: Strigiformes) nas Estações Ecológica de Jataí e Experimental de Luiz Antônio, SP. Estação Ecológica de Jataí, 1, 303--315.

Novelli, R., Takase, E., \& Castro, V. 1988. Estudo das aves mortas por atropelamento em um trecho da rodovia BR-471, entre os distritos da Quinta e Taim, Rio Grande do Sul, Brasil. Revista Brasileira de Zoologia, 5(3), 441--454. DOI: 10.1590/S0101-81751988000300009

Pautasso, A. A. 2006. Dieta del lechuzón orejudo (Asio clamator) en el centro y este de la provincia de Santa Fe, Argentina. Ornitologia Neotropical, 17(2), 289--293.

Peters, F. B., Roth, P. R. O., Jeronimo, A. A., Pereira, M. S., Poerschke, F., Machado, L. F., \& Christoff, A. U. 2009. predação de Lepus europaeus (Lagomorpha: Leporidae) por Bubo virginianus (Strigiformes: Strigidae) no sul do Brasil. Biodiversidade Pampeana, 7(1), 31--34.

Ramos, C. C. O., Benedito E., \& Zawadzki C. H. 2011. Dieta e conteúdo calórico de aves atropeladas na região central do estado do Paraná, Brasil. Biotemas, 24(4), 153--170. DOI: 10.5007/2175-7925.2011v24n4p153

Rocha, R. G., Ferreira, E., Leite, Y. L. R., Fonseca, C., \& Costa, L. P. 2011. Small mammals in the diet of Barn owls, Tyto alba (Aves: Strigiformes) along the mid-Araguaia River in central Brazil. Zoologia, 28(6), 709--716. DOI: 10.1590/S198446702011000600003

Scheibler, D. R., \& Christoff, A. U. 2004. Small 
mammals in the diet of barn owls (Tyto alba) in agroecosystems of southern Brazil. Ornitologia Neotropical, 15(1), 65--70.

Sick, H. 1997. Ornitologia brasileira. Rio de Janeiro: Nova Fronteira: p. 862

Teta, P., Hercolini, C., \& Cueto, G. 2012. Variation in the diet of Western Barn Owls (Tyto alba) along an urban-rural gradient. The Wilson Journal of Ornithology, 124(3), 589--596. DOI: 10.1676/11173.1

Tomazzoni, A. C., Pedó, E., \& Hartz, S. M. 2004. Food habitats of Great Horned Owls (Bubo virginanus) in the breeding season in Lami Biological Reserve Southern Brazil. Ornitologia Neotropical, 15(2), 279--282.

Trejo, A, Kun, M., Sahores, M., \& Seijas, S. 2005. Diet overlap and prey size of two owls in the forest-steppe ecotone of southern Argentina. Ornitologia Neotropical, 16(4), 539--546.

Triplehorn, C. A., \& Johnson, N. F. 2011. Estudo dos insetos: tradução da 7th ed de Borror and Delong's introduction to the study of insects. São Paulo: Cengage Learning: p. 809.

Vieira, W. L. S., Bezerra, D. M. M., Vieira, K. S., Santana, G. G., Montenegro, P. F. G. P., \& Alves, R. R. N. 2015. Megascops choliba (Strigiformes: Strigidae) predation on Scinax $x$-signatus (Anura: Hylidae) in the semiarid, Northeastern Brazil. Herpetology Notes, 8(1), 275--276.

Zilio, F. 2006. Dieta de Falco sparverius (Aves: Falconidae) e Athene cunicularia (Aves: Strigidae) em uma região de dunas no sul do Brasil. Revista Brasileira de Ornitologia, 14(4), 379--392.

Zilio, F., Zucatti, B., \& Scheibler, D. R. 2018. Diet of Long-tufted Screech-Owl in a southern Brazilian agroecosystem. Journal of Raptor Research, 52(1), 115--118. DOI: 10.3356/JRR-16-107.1

Zúñiga, A. H., Fuenzalida, V., \& Sandoval, R. 2018. Hábitos alimentarios de la lechuza blanca Tyto alba en un agroecosistema del centro-sur de Chile. Ecología en Bolivia, 53(1), 7--15.

Submitted: 24 May 2019

Accepted: 29 September 2019

Published online: 09 October 2019

Associate Editor: Sara Miranda Almeida 